\title{
EL CRIBADO SISTEMÁTICO SOBRE VIOLENCIA DE GÉNERO DURANTE EL EMBARAZO. UNA CUESTIÓN ESPECIALMENTE IMPORTANTE EN TIEMPOS DE COVID
}

Systematic Screening for Gender-Based Violence during Pregnancy. A Pressing Issue in Times of COVID-191

María Analía Gómez-Fernández ${ }^{1}$, Carme Homs-Mir², Dolores Gómez-Martín², Sara Merayo-Fernández ${ }^{1}$

Autora correspondencia: María Analía Gómez-Fernández

Correo electrónico: analiagomez2@yahoo.es

1. Servicio de Salud de Castilla y León. (León, España)

2. Instituto Catalán de la Salud. (Barcelona, España)

Recibido: 13/02/2021 Aceptado 30/07/2021

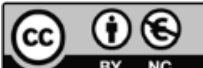

${ }^{1}$ La investigación que ha dado lugar a estos resultados ha sido financiada por el proyecto Recercaixa (2016ACUP 0083) y financiada parcialmente por la Fundación Enfermería y Sociedad en el marco de las Ayudas a la Investigación Enfermera (PR-050/16). 


\section{RESUMEN}

OBJETIVO. Conocer la experiencia de las matronas de atención primaria en el cribado sistemático de la violencia de género $(\mathrm{VG})$ durante el embarazo con un cuestionario validado y su utilidad. Metodología. Estudio cualitativo con un enfoque fenomenológico interpretativo. Tras la experiencia de cribar de forma sistemática durante cuatro meses, se hicieron dos grupos focales con las doce matronas que prestan asistencia en dos centros de atención primaria de L'Hospitalet de Llobregat (Barcelona). RESULTADOS. Aplicar de forma sistemática el cuestionario Index of Spousal Abuse, previa formación para su aplicación y para el abordaje de los casos de VG, aumenta las habilidades, los conocimientos y la seguridad de las matronas para detectar y abordar la VG durante el embarazo. A su vez, incrementa la detección de casos de VG. CONCLUSIONES. Es necesario incorporar el cribado sistemático de la VG durante el seguimiento del embarazo.

Palabras clave: violencia de género; embarazo; matronas; cribado sistemático; investigación cualitativa.

\section{ABSTRACT}

OBJECTIVE. To understand primary care midwives' experience with the use of a validated survey for systematic screening for intimate partner violence during patient pregnancy and the usefulness of said screening. METHOD. A qualitative study with an interpretative phenomenological approach performed subsequently to systematic screening experiences had over four months. Focus groups of twelve midwives provided assistance in two primary care centres in L'Hospitalet de Llobregat (Barcelona, Spain). RESULTS. The systematic use of the Index of Spousal Abuse survey with prior training on its use as well as on approaching cases of intimate partner violence enhance midwives' knowledge levels, skills, and confidence in relation to detecting and approaching intimate partner violence situations during a pregnancy. Systematic screening concurrently increases rate at which cases of intimate partner violence are detected. CONCLUSIONS. Systematic screening for intimate partner violence must become an integral part of pregnancy monitoring.

Keywords: Intimate Partner Violence; Pregnancy; Midwives; Mass Screening; Qualitative Methodology. 


\section{INTRODUCCIÓN}

La violencia de género (VG) fue definida por las Naciones Unidas (1993) como «todo acto de agresión a la mujer basado en la pertenencia al sexo femenino que tenga o pueda tener como resultado un daño o sufrimiento físico, sexual o psicológico, así como las amenazas de tales actos, coacción o privación arbitraria de la libertad, tanto si se producen en la vida pública como en la vida privada». La Organización Mundial de la Salud (OMS) define la VG como un problema de salud pública (2013) porque afecta a la salud física de las mujeres, a causa de las lesiones directas o por somatización y/o a la salud mental, provocando depresión, ansiedad, etc.; y porque es muy prevalente (Ministerio de Sanidad y Consumo, 2012). A nivel mundial, el 30\% de las mujeres que han tenido pareja, han experimentado algún tipo de VG (OMS, 2017). Si, además, ocurre durante el embarazo, las graves consecuencias que tiene para la salud de la mujer se amplían por la posibilidad de desencadenar complicaciones en la gestación, parto y posparto, tales como un mayor riesgo de aborto, retraso de crecimiento intrauterino, parto prematuro, depresión posparto o incluso muerte neonatal (Ministerio de Sanidad y Consumo, 2012; Pastor-Moreno, Ruiz-Pérez y Rucci-Cabello, 2018). En la actual situación de estado de alarma por la pandemia de la COVID19, se añade el aislamiento en los hogares y el cierre de muchos servicios (Ministerio de Igualdad, 2020a). La convivencia obligatoria que supone para las familias la cuarentena por el coronavirus ha aumentado el riesgo de sufrir agresiones en el caso de las mujeres víctimas de VG, que se ven obligadas a compartir constantemente el espacio del hogar con sus agresores (Ministerio de Igualdad, 2020b). En España, en marzo de 2020, las llamadas al 016 (teléfono de información y de asesoramiento en VG) aumentaron un 10,5\% y las consultas online un $182,93 \%$ con respecto al mes de marzo del año anterior (Delegación del Gobierno para la Violencia de Género, 2020). Los estados de confinamiento reúnen varios factores que potencian el riesgo de sufrir VG: más tiempo de convivencia víctima-agresor; mayor control sobre la víctima; más dificultad para recibir ayuda externa; agresiones más invisibilizadas y más dificultad para acudir a los centros de asistencia (Unidad de Igualdad. Universidad de Málaga, 2020). En este sentido, la ONU (Naciones Unidas, 2020) ha realizado un llamamiento mundial para actuar frente al repunte de la VG durante la pandemia y en situaciones de aislamiento.

Por otro lado, el cribado sistemático sobre VG desde los servicios de salud ha sido motivo de controversia. Aunque hay literatura que lo apoya (Bourey, et al., 2015; Ghandour, Campbell y Lloyd, 2015), una revisión Cochrane (O’Doherty et al., 2014) 
concluye que se necesitan más estudios para recomendarlo. Sin embargo, según las directrices de la OMS para la práctica clínica en respuesta a la violencia de pareja y a la violencia sexual contra las mujeres (OMS y OPS, 2014), en caso de que la mujer presente un trastorno mental, sea seropositividad al VIH o esté embarazada, por su mayor vulnerabilidad, sí se recomienda. El cribado sistemático durante el seguimiento del embarazo y posparto es defendido también por el Colegio Estadounidense de Obstetras y Ginecólogos (2017) y la Sociedad Española de Ginecología y Obstetricia (2017). En esa misma línea, la OMS (2014) indica que indagar sobre VG durante las consultas prenatales puede dar mejores resultados para la salud de la mujer por la posibilidad de seguimiento durante la atención prenatal. Siempre se debe preguntar sobre VG de una forma segura para la mujer, es decir de forma privada y asegurando la confidencialidad (UNFPA and WAVE, 2014). Respecto a la forma de preguntar, el «Protocolo común para la actuación sanitaria ante la violencia de género» del Ministerio de Sanidad y Consumo (2012) muestra algunos ejemplos de preguntas abiertas, tales como «Durante el embarazo a veces, se agudizan los problemas o conflictos de pareja ¿se encuentra usted en esta situación?, ¿cómo vive su pareja el embarazo? También se han desarrollado y validado diversos cuestionarios con el fin de identificar a las víctimas de la VG (OMS, 2013), pero sigue habiendo una falta de consenso con respecto cual puede ser el más efectivo (Chisholm et al., 2017).

Es fundamental documentar la experiencia de las matronas en el cribado sistemático de la VG durante el embarazo y su utilidad, siguiendo de esta forma una de las Estrategias Nacionales para la Erradicación de la Violencia contra la Mujer 2013-2016 del Ministerio de Sanidad, Servicios Sociales e Igualdad (2013), donde se recomienda identificar y difundir buenas prácticas en la prevención y atención de la VG.

El presente trabajo forma parte de un proyecto de investigación más amplio cuyo objetivo principal fue promover cambios que mejoren la detección y abordaje de la VG durante el embarazo por parte de las matronas. Dicho proyecto se fundamentó en el uso de la metodología de la investigación acción participativa (IAP) (Kemmis y McTaggart, 1988) y se ha desarrollado en diferentes fases: en un primer momento se procedió a analizar la situación y a detectar necesidades, seguidamente se elaboraron e implementaron las estrategias de acción, para finalmente evaluarlas. En el presente artículo se presentan los resultados de la evaluación de una de las estrategias de acción implementadas y tiene como objetivo conocer la experiencia de las matronas de atención 
primaria en el cribado sistemático de la VG durante el embarazo con un cuestionario validado y su utilidad.

\section{METODOLOGÍA}

\section{Diseño}

Estudio cualitativo con enfoque fenomenológico interpretativo, de acuerdo con Mackey (2005), con el propósito de comprender las experiencias vividas por las matronas respecto al fenómeno del cribado sistemático de la VG durante el embarazo.

\section{Ámbito}

Dos centros de atención primaria (AP) con titularidad pública (Just Oliveras y Ronda Torrassa) en L'Hospitalet de Llobregat (Barcelona), con una población asignada de 257.349 habitantes (Generalitat de Catalunya, 2017). En los centros participantes 12 matronas son las encargadas de realizar el seguimiento del embarazo a una media de 2.100 mujeres al año (según datos de la propia institución). En caso de existir algún problema de salud, el seguimiento del embarazo se realiza de forma conjunta con los ginecólogos/as.

\section{Recogida de datos}

Se presentó a las matronas el estudio y se solicitó su colaboración: todas desearon participar. Para el desarrollo de la IAP, se procedió a analizar la situación y a detectar necesidades, mediante la realización de entrevistas individuales semiestructuradas a las mismas (Gómez-Fernández, Goberna-tricas y Payà-Sánchez, 2019). Seguidamente se procedió a la elaboración e implementación de las estrategias de acción. Una de ellas fue cribar de forma sistemática sobre VG durante el embarazo con un cuestionario validado. Para elegir el cuestionario más oportuno, se procedió a la revisión de los cuestionarios validados de detección de VG (Gómez-Fernández, Goberna-Tricas, y Payà-Sánchez, 2018), seleccionado el cuestionario Index of Spouse Abuse (ISA) en su versión adaptada al español, por sus excelentes propiedades psicométricas ( $\alpha$ Cronbach 0.98) (PlazaolaCastaño, et al., 2009) y porque indaga a través de 30 preguntas sobre violencia psicológica, física, sexual y comportamientos de control. Antes de proceder al uso del cuestionario, las matronas acudieron a una actividad formativa con una duración de 12 horas donde se trataron temas como indicadores de sospecha, empleo de cuestionarios, 
circuitos de atención, acompañamiento psicosocial a la mujer, habilidades comunicativas y aspectos éticos (Payà-Sánchez, et al., 2019). Durante cuatro meses (de marzo a junio de 2018) las matronas cribaron de forma sistemática sobre VG aplicando el cuestionario ISA a las mujeres a medida que acudían a las visitas de seguimiento del embarazo, previo consentimiento informado (cumplimentaron el cuestionario 432 gestantes). Seguidamente (entre julio-diciembre de 2018), se procedió de la evaluación de la estrategia de acción, desde una perspectiva fenomenológica cuyos resultados se presentan en este trabajo. Se concretó, vía correo electrónico, día y hora para realizar dos grupos focales con las matronas, uno en cada centro (al primero acudieron las 7 matronas que prestan asistencia en Ronda Torrassa y al segundo las 5 matronas que prestan asistencia en Just Oliveras). Ambos grupos fueron dirigidos por la misma investigadora MAGF. Cada grupo focal fue grabado y la duración promedio fue de sesenta minutos, se realizaron notas de campo durante todo el proceso. Se utilizó un guion de preguntas abiertas que actuaron como pregunta estímulo (Tabla 1). Se tomaron notas de campo durante todo el proceso. No se repitió ningún grupo focal, puesto que se había respondido a lo que se solicitaba. Paralelamente, los datos obtenidos en el segundo grupo focal fueron muy similares al primero; por lo tanto, se consideró que la recopilación de datos había alcanzado el punto de saturación. Conjuntamente, se comparó el número de casos detectados antes y tras las acciones implementadas.

Tabla 1. Guía temática de las entrevistas ¿Cómo ha sido vuestra experiencia con la aplicación del cuestionario ISA? ¿Según vuestra experiencia creéis que el cuestionario ISA puede ser una herramienta útil?

¿Ha influenciado en la manera de detectar la VG el uso del cuestionario?

¿Qué reacciones habéis tenido por parte de las mujeres cuando les proponías participar el estudio y al cumplimentar el cuestionario?

\section{Participantes}

Las doce matronas de atención primaria de L’Hospitalet de Llobregat. 


\section{Análisis}

Se realizó una transcripción literal de las grabaciones de los grupos focales. Los textos fueron enviados vía correo electrónico a las participantes, por si deseaban hacer alguna aclaración o modificación. Se realizó en análisis de los datos siguiendo un enfoque inductivo, y de acuerdo a las recomendaciones de Taylor and Bogdan (1987): 1) Descubrimiento 2) Codificación de los datos y 3) Relativización. Las tres investigadoras identificaron de forma independiente los códigos iniciales antes de reunirse en diversas ocasiones para consensuar el sistema de categorías y triangular la aplicación de los códigos. Cabe señalar que se encontró un gran consenso. Con el apoyo del programa Atlas-Ti v.7.5.10 se procedió a sistematizar la codificación, estableciendo las relaciones entre categorías y subcategorías.

\section{Consideraciones éticas}

Para realizar el estudio se siguieron las Recomendaciones éticas y de seguridad para la investigación sobre la violencia doméstica contra las mujeres de la Organización Mundial de la Salud (2001) y se cumplió con la Ley Orgánica 3/2018 de Protección de Datos Personales y garantía de los derechos digitales (BOE Núm. 294, 6 de diciembre de 2018). Previamente a la realización de la investigación, se obtuvo la aprobación del proyecto por parte de la Comisión de Bioética de la Universidad de Barcelona (IRB00003099) y del Comité Ético de Investigación Clínica del IDIAP Jordi Gol (P15/129). Se facilitó a las participantes de forma verbal y escrita información de los objetivos y del procedimiento del estudio. Cuando aceptaron participar, se solicitó su consentimiento por escrito, asegurándoles la confidencialidad y anonimato (se les entregó una copia de dicho consentimiento). Las dudas fueron atendidas y se puso a su disposición datos de contacto de la investigadora principal.

\section{RESULTADOS}

Se entrevistó a un total de doce matronas, el total de las matronas de L'Hospitalet, cuyas características pueden observarse en la Tabla 2 (identificadas como m1-m12). Todas son mujeres y tienen una relación laboral permanente con la institución. Las doce desempeñan tareas asistenciales, dos de ellas combinándolas con funciones de gestión. Respecto a los años de experiencia como matronas en AP, existe un amplio rango de entre 2 y 30 años de experiencia, que no guarda siempre relación directamente proporcional con la edad. 
De hecho, si bien actualmente todas las matronas se dedican a tiempo completo a su trabajo en AP, todas tienen experiencia previa a nivel hospitalario en el área maternoinfantil.

Tabla 2. Características de las matronas

\begin{tabular}{|c|c|c|c|c|}
\hline Matrona & Edad & Enfermería $^{a}$ & Matrona $^{b}$ & $\begin{array}{c}\text { Matrona en } \\
\text { atención primaria }\end{array}$ \\
\hline m1 & 60 & 1977 & 1979 & 15 \\
\hline m2 & 59 & 1978 & 1981 & 17 \\
\hline m3 & 59 & 1978 & 1982 & 26 \\
\hline m4 & 58 & 1982 & 1984 & 20 \\
\hline m5 & 56 & 1982 & 1985 & 24 \\
\hline m6 & 56 & 1982 & 1986 & 27 \\
\hline m7 & 55 & 1983 & 1986 & 30 \\
\hline m8 & 53 & 1985 & 1987 & 14 \\
\hline m9 & 53 & 1985 & 1996 & 20 \\
\hline m10 & 41 & 1996 & 1998 & 13 \\
\hline m11 & 39 & 1998 & 2013 & 2 \\
\hline m12 & 34 & 2003 & 2008 & 8 \\
\hline
\end{tabular}

a) año de finalización de los estudios de Enfermería;

b) año de finalización de los estudios de Matrona;

c) años de experiencia como matrona en atención primaria.

Durante la fase de análisis de la situación, las matronas revelaron que solo preguntaban sobre VG en caso de sospechar que la mujer la sufriera. De las doce matronas entrevistadas, cuatro no recordaban haber detectado ningún caso de VG durante su ejercicio profesional en AP. Las ocho matronas restantes afirmaban haber detectado entre uno y cuatro casos, casi siempre de violencia física y ninguno durante el año 2016. Sin embargo, tras la asistencia a la actividad formativa y aplicando el cuestionario ISA de forma sistemática (en un periodo de solo cuatro meses), todas las matronas detectaron entre 2 y 9 casos.

A continuación, se presenta la información más relevante en base a la experiencia de las matronas en el cribado sistemático de la VG durante el embarazo y su valoración del cuestionario. 


\section{Las vivencias de las matronas}

\section{El cuestionario}

La mayoría de las matronas consideran el cuestionario ISA como útil. El hecho de que el cuestionario deba ser autocumplimentado por la mujer, según la mayoría de las matronas es una ventaja:

Creo que es mejor que sea la propia mujer la que rellene [...] que lo pueda leer y lo pueda reflexionar y pensar antes de marcar. A veces cuando alguien te pregunta, contestas quizás lo que crees que es correcto contestar. (m1)

A pesar de que el cuestionario ISA se cumplimenta en unos cinco minutos, desde la perspectiva de algunas matronas, podría ser útil incorporar un cuestionario con menos preguntas, incluso una matrona alude a la posibilidad de no acortar el número de preguntas sino las opciones de respuesta, con el objetivo final igualmente de optimizar el tiempo de la visita:

[...] menos opciones de puntuación, porque igual se ponen a pensar entre 2 y 3 ¿no? Igual eso les hace pararse más a pensar, más que la pregunta, igual les hace pararse a pensar que puntuación le ponen. Porque o son los extremos que lo tiene muy claro o lo otro le hace detenerse más. $(\mathrm{m} 11)$

\section{Las reacciones de las mujeres}

De forma unánime afirman que las mujeres no se han sentido ofendidas y se han mostrado muy colaboradoras para cumplimentar el cuestionario. La mayoría de las matronas muestran asombro de la sinceridad de las mujeres a la hora de cumplimentar el cuestionario:

A las mujeres no les ha costado nada participar, como si estuvieran esperando que alguien les diera la opción para poder expresarse [...] porque yo pensaba al principio que las mujeres se quedarían muy cortadas, pero luego las he visto muy decididas a responder. (m13)

Se desprende de sus reflexiones que incluso en los casos en que las mujeres no se encuentran en una situación violenta, con la aplicación del cuestionario ISA se genera un espacio para hablar de VG y sensibilizar a la población.

Alguna mujer te decía: hace unos años me pasó esto exactamente y hasta que me di cuenta y dejé a esa pareja, pues pasó mucho tiempo. (m12) 


\section{Los indicadores de sospecha}

Las matronas verbalizan que, en la mayoría de los casos detectados con el cuestionario ISA, no tuvieron en ningún momento la sospecha de que la mujer se pudiera encontrar en una situación de VG:

Había ido al hospital unas semanas antes [a causa de una agresión física por parte de su pareja] y no me decía nada, si no le paso el cuestionario, no me entero, no me lo dice. (m4)

\section{Emociones de las matronas}

Escuchar los relatos de violencia no deja indiferente a las matronas, las cuales refieren sentimientos de tristeza y rabia que aparecen especialmente cuando, en sucesivas visitas de seguimiento del embarazo, se enfrentan a que esté presente la pareja:

Rabia, sí, me siento rabiosa, porque cuando lo sabes [...]. Yo creo que nosotras ya nos los miramos con otros ojos. (m9)

\section{El aprendizaje generado}

\section{La seguridad adquirida}

Tras la formación recibida y la experiencia de aplicar un cuestionario de forma sistemática, las revelaciones de las matronas evidencian que se ha generado aprendizaje y se han disipado algunos de los miedos que afloraban en la fase de análisis de la situación, donde las matronas consideraban que las mujeres se podrían ofender al preguntarles por VG (o no querrían revelar su situación) o tenían miedo a no saber abordar la situación si la mujer revelara una situación de violencia

[...] si preguntas, te encuentras, si te encuentras ¿qué haces? Entonces lo primero que tienes que saber es ¿cómo ayudarla? ¿Dónde derivarla? Porque si no lo sabes, no preguntes. Entonces ahora sí lo sé [...] Yo ahora me siento más cómoda. $(\mathrm{m} 1)$

\section{La implicación para la práctica}

Según las matronas, con el cribado sistemático han desarrollado destrezas y tienen más presente la posibilidad de que una mujer se pueda encontrar en una situación de VG:

Tomas más conciencia. Incluso en mujeres que te sorprende también, que haya dado un resultado determinado, cuando no te lo esperabas. (m6)

[...] empiezas a tener cierta agudeza para detectar mujeres que no lo expresan, pero tu percibes que algo pasa. (m4) 


\section{DISCUSIÓN}

Las matronas consideran que, tras la experiencia de preguntar sistemáticamente por la VG, se sienten más seguras y han mejorado sus conocimientos, puesto que la práctica clínica es la mejor forma de afianzar los conocimientos teóricos (Hinderliter, et al., 2003). Expresan que las mujeres aceptaban con naturalidad ser preguntadas sobre VG (Stöckl et al., 2013) y comentan que, según su experiencia, en caso de encontrarse en una situación de violencia, tenían la percepción de que las mujeres estaban deseando que alguien les preguntara (OMS, 1998), igual que en Kataoka y Imazeki (2018), donde las gestantes valoraban como oportuno realizar preguntas sobre VG durante el control gestacional, incluidas las que revelaban violencia. Además, en su conjunto, la aplicación sistemática del cuestionario ISA, según las matronas, ha generado un espacio en las consultas para sensibilizar sobre VG e, igual que en Williams et al. (2017), puede contribuir a desmontar mitos. Algunas matronas aludían a la posibilidad de incorporar un cuestionario más breve que el ISA. En este sentido, la Guía Práctica Clínica: Actuación en salud mental con mujeres maltratadas por su pareja del Servicio Murciano de Salud (2011) recomienda aplicar un cuestionario de cribado, el WAST en su versión reducida (de dos preguntas) $y$, en el supuesto de que el resultado sea positivo o que el sanitario sospeche que la mujer se encuentra en una situación de violencia, confirmarlo con una entrevista clínica y/o cuestionario ISA. Algunos protocolos de seguimiento de embarazo se han decantado por un cuestionario en concreto. Este sería el caso del Protocolo de seguimiento del embarazo en Cataluña publicado en junio del 2018 (el cual no estaba en vigor durante la elaboración de este trabajo), que indica el uso del Partner Violence Screen (PVS). El PVS es un cuestionario de cribado que consta de tres preguntas (Generalitat de Catalunya, 2018). Sin embargo, Velasco et al. (2014) aplicaron a todas las gestantes de su estudio el cuestionario AAS (de cinco preguntas) y el ISA, obteniendo una prevalencia de VG del $7,7 \%$ y $21,3 \%$ respectivamente. Esos resultados llevan a reflexionar sobre la posibilidad de que con los cuestionarios más breves se optimice el tiempo de la consulta pero se disminuyan los casos detectados.

Cuatro de las doce matronas entrevistadas no habían detectado ningún caso de violencia en el embarazo en su trayectoria profesional en AP. Ello podría constituir un indicio para considerar que la VG durante el embarazo estaba infradetectada (HerreraGómez y Martín-Martínez, 2016). Sin embargo, igual que en O’Doherty et al. (2014), al incorporar la aplicación del cuestionario de forma sistemática al control gestacional, ha 
aumentado de forma muy significativa la detección de la VG con respecto a la atención habitual, donde se preguntaba solo en casos de sospecha. En ese mismo sentido, un estudio en un servicio de urgencias canadiense comparó la utilización de un cuestionario de cinco preguntas sobre la $\mathrm{VG}$, con respecto a la atención habitual de urgencias, y encontró que el cuestionario aumentaba el porcentaje de detección desde 0,4\% a 14\% (Morrison, Allan y Grunfeld, 2000). Durante el embarazo la detección puede aumentar hasta en cuatro veces con el uso sistemático de cuestionario de detección (O’Doherty et al., 2014).

En la mayoría de los casos detectados con el cuestionario ISA, las matronas no sospechaban que las mujeres estuvieran en una situación de violencia, lo que refuerza la estrategia del cribado sistemático, es decir, preguntar a todas las mujeres independientemente de que se detecten o no indicadores de sospecha (Chisholm et al., 2017; Olive, 2007). Todahl y Walters (2011) señalan que preguntar sobre VG a todas las mujeres, además de generar mayor experiencia en el profesional, evita la sensación por parte de la paciente de ser señalada. En la situación actual de pandemia, donde se ha reorganizado el seguimiento del embarazo para minimizar la exposición de las gestantes a los sistemas sanitarios (Servei Català de la Salut, 2020), no debemos perder la oportunidad de indagar sobre este grave problema de salud en las visitas presenciales. Siendo imprescindible que los servicios sanitarios y sociales continúen sus obligaciones de atención a estas mujeres (OMS, 2020). Como es indiscutible, las ventajas del cribado sistemático solo pueden obtenerse si este se complementa con protocolos que incorporen el apoyo a las víctimas en la práctica habitual (OMS, 2013; Stöck1, 2014) y una formación previa adecuada (González Muñoz, Durán Flores y González Rubio, 2019). Conjuntamente, en el contexto de la pandemia, el Ministerio de Igualdad, a través de la Delegación de Gobierno contra la Violencia de Género, ha impulsado un Plan de Contingencia contra la violencia de género que se ha ido concretando en diversas actuaciones y medidas específicas (Ministerio de Igualdad, 2020b).

Cabe señalar como limitación de este estudio que, al ser cualitativo centrado en el contexto de las matronas de un ámbito concreto, los resultados solo pueden ser transferibles a otros contextos que guarden semejanza con el estudiado. 


\section{CONCLUSIONES}

Aplicar de forma sistemática el cuestionario Index of Spousal Abuse previa formación para su aplicación y para el abordaje de los casos de VG se ha mostrado como útil en el ámbito asistencial. Puesto que, según la experiencia de las matronas, aumenta sus habilidades, sus conocimientos y su seguridad para detectar y abordar la VG durante el embarazo. En su conjunto, incrementa la detección de casos de VG, mostrándose, por tanto, la necesidad de incorporar el cribado sistemático de la VG durante el seguimiento del embarazo, lo cual se hace todavía más importante en tiempos de pandemia.

\section{AGRADECIMIENTOS}

A las matronas y a las mujeres que han aceptado participar en este trabajo. A la Universidad de Barcelona, al Instituto Universitario de Investigación en Atención Primaria Jordi Gol y al Instituto Catalán de la Salud por su apoyo en esta investigación. 


\section{BIBLIOGRAFÍA}

BOE-Boletín Oficial Del Estado. Ley Orgánica 3/2018, de Protección de Datos Personales y garantía de los derechos digitales. 6 de diciembre de 2018, núm. 294.

BOUREY, C., et al. Systematic review of structural interventions for intimate partner violence in low- and middle-income countries: organizing evidence for prevention. En: BMC Public Health. 2015, vol. 15, núm. 1, pp. 1-18.

Chisholm, C.; Bullock, L.; Ferguson, J. Intimate partner violence and pregnancy: screening and intervention. En: American Journal of Obstetrics and Gynecology. 2017, vol. 217, núm. 2, pp. 145-149.

Delegación del Gobierno para la Violencia de GÉnero. Portal Estadístico Violencia de Género. 2020. Disponible en: https://bit.ly/322ip9o.

Generalitat de Catalunya. Instituto de Estadística de Cataluña. 2017. Disponible en: https://www.idescat.cat.

Generalitat de Catalunya. Protocol de Seguiment d'Embaràs a Catalunya. 2018. Disponible en: https://bit.ly/3tvusay.

Ghandour, R.; CAMPBell, J.; Lloyd, J. Screening and Counseling for Intimate Partner Violence: A Vision for the Future. En: Journal of Women's Health. 2015, vol. 24, núm. 1, pp. 57-61.

Gómez-Fernández, M. A.; Goberna-Tricas, J.; PAyÀ-SÁnchez, M. Characteristics and clinical applicability of the validated scales and tools for screening, evaluating and measuring the risk of intimate partner violence. Systematic literature review (2003-2017). En: Aggression and Violent Behavior. 2018, vol. 44, pp. 57-66.

GómeZ-Fernández, M. A.; Goberna-Tricas, J.; PAyÀ-SÁnCheZ, M. El saber experiencial de las matronas de Atención Primaria en la detección de violencia de género durante el embarazo. Estudio cualitativo. En: Enfermería Clínica. 2019, vol. 29, núm. 6, pp. 344-351.

GonzÁlez Muñoz, P. F.; DurÁn Flores, M. L.; GonzÁlez Rubio, M. J. Conocimientos, actitudes y opiniones sobre violencia de género en el profesional de enfermería de atención primaria. En: Enfermería Comunitaria. 2019, vol. 15.

Herrera-Gómez, A.; Martín-Martínez, F. J. Detección precoz de la violencia de género: hacia el diagnóstico biológico Cuando la innovación se convierte en rutina. En: Index Enferm. 2016, vol. 25, núm. 4, pp. 229-230.

Hinderliter, D., et al. The Effect of Intimate Partner Violence Education on Nurse Practitioners' Feelings of Competence and Ability to Screen Patients. En: Journal of Nursing Education. 2003, vol. 42, núm. 10, pp. 449-454.

KATAOKA, Y.; IMAZEKI, M. Experiences of being screened for intimate partner violence during pregnancy: a qualitative study of women in Japan. En: BMC Women's Health. 2018, vol. 18, núm. 75, pp. 1-9. 
Kemmis, S.; Mctaggart, R. Cómo planificar la investigación-acción. Barcelona: Laertes, 1992

MACKEY, S. Phenomenological nursing research: methodological insights derived from Heidegger's interpretive phenomenology. International Journal of Nursing Studies. En: International Journal of Nursing Studies. 2005, vol. 42, núm. 2, pp. 179-186.

MinisTERIO DE IGUALDAD. Estamos contigo. La violencia de género la paramos unidas. Guía de actuación para mujeres que estén sufriendo violencia de género en situación de permanencia domiciliaria derivada del estado de alarma por COVID 19. 2020a. [Consulta:13 de febrero de 2021]. Disponible en: https://bit.ly/3GCkoAl.

Ministerio DE IGUALDAD. La perspectiva de género, esencial en la respuesta a la COVID-19. 2020b. [Consulta: 11 de febrero de 2021]. Disponible en: https://bit.ly/3qyePgU.

Ministerio de SANidAd, SERVicios Sociales E IGUALDAD. Estrategia Nacional para la erradicación de la violencia contra la mujer (2013-2016). 2013. [Consulta: 10 de enero de 2019]. Disponible en: https://bit.ly/3A2gX3s.

MinISTERIO DE SANIDAD Y CONSUMO. Protocolo común para la actuación sanitaria ante la violencia de género. 2012. [Consulta: 8 de agosto de 2019]. Disponible en: https://bit.ly/3fsf32D.

Morrison, L.; Allan, R.; GRUNFELD, A. Improving the emergency department detection rate of domestic violence using direct questioning. En: The Journal of Emergency Medicine. 2000, vol. 19, núm. 2, pp. 117-124.

NACIONES UNIDAS. Declaración sobre la eliminación de la violencia contra la mujer. Resolución de la Asamblea General. 1993. [Consulta: 9 de agosto de 2019]. Disponible en: https://bit.ly/3I53kTO.

NACIONES UnidAS. Declaración del Secretario General sobre la lucha contra la violencia de género y el COVID-19. 2020. [Consulta: 5 de desembre de 2020]. Disponible en: https://bit.ly/3qxfE9B.

O'DoherTy, L. J., et al. Screening women for intimate partner violence in healthcare settings: abridged Cochrane systematic review and meta-analysis. En: BMJ. 2014, vol. 348. Disponible en: https://www.bmj.com/content/348/bmj.g2913.full

Olive, P. Care for emergency department patients who have experienced domestic violence: a review of the evidence base. En: Journal of Clinical Nursing. 2007, vol. 16, núm. 9, pp. 1736-1748.

OMS - ORGANIZACIÓN Mundial DE LA SALUd. Violencia contra la mujer Un tema de salud prioritario. Ginebra: OMS, 1998.

OMS - ORgAnizAción Mundial DE SAlud. Dando prioridad a las mujeres: Recomendaciones éticas y de seguridad para la investigación sobre la violencia doméstica contra las mujeres. Ginebra: OMS, 2001. [Consulta: 22 de octubre de 2018]. Disponible en: https://bit.ly/3FyN5Nd. 
OMS - ORganización Mundial de SALUd. Global and regional estimates of violence against women: prevalence and health effects of intimate partner violence and nonpartner sexual violence. Ginebra: OMS, 2013. [Consulta: 4 de abril de 2019]. Consulta en: https://bit.ly/3KsLCvR.

OMS - ORGANIZACIÓN MuNDIAL DE SALUD. Violence against women. Intimate partner and sexual violence against women. Ginebra: OMS, 2017. [Consilta: 18 de abril de 2019]. Disponible en: http://www.who.int/mediacentre/factsheets/fs239/enOMS -

OMS - Organización Mundial de la SAlud. Prevención de la violencia: la evidencia. Ginebra: OMS, 2013. [Consulta: 1 de setembre de 2018]. Disponible en: https://bit.ly/3fqMmTT.

OMS - ORgANIZACIÓn Mundial DE LA SALUd. Declaración entre organismos sobre la violencia contra las mujeres y las niñas en el contexto de la COVID-19. Ginebra: OMS, 2020. [Consulta: 15 de diciembre de 2020]. Disponible en: https://bit.ly/3quenYG.

OMS - ORganización Mundial de SALud; OPS - ORganización PARAMERICANA DE LA SALUD. Respuesta a la violencia de pareja y a la violencia sexual contra las mujeres. Directrices de la OMS para la práctica clínica y las políticas. Ginebra: OMS, 2014. Disponible en: https://bit.ly/3fwbKYc.

Pastor-Moreno, G.; Ruiz-PÉrez, I.; Rucci-CABello, I. Historia de Violencia y Violencia de Compañero Íntimo en Resultados en salud. En: Index Enferm. 2018, vol. 27, núm. 4, pp. 191-195.

PAYÀ-SÁNCHEZ, M., et al. Formación para la detección de la violencia de género durante el embarazo: evaluación de una propuesta contextualizada. MUSAS. Revista de investigación en Mujer, Salud y Sociedad. 2019, vol. 4, núm. 1, pp. 22-38. Disponible en: https://doi.org/10.1344/musas2019.vol4.num1.2.

Plazaola-Castaño, J., et al. Validation of the Spanish Version of the Index of Spouse Abuse. En: Journal of Women's Health. 2009, vol. 18, núm. 4, pp. 499-506.

Servei Català de la Salut. Guia d'actuació enfront de casos d'infecció pel nou coronavirus SARS-CoV-2 en dones embarassades $i$ nadons Document operatiu. 2020. Disponible en: https://canalsalut.gencat.cat/web/.content/_A-Z/C/coronavirus2019-ncov/material-divulgatiu/guia-actuacio-embarassades.pdf.

Servicio Murciano de Salud. Guía Práctica Clínica. Actuación en salud mental con mujeres maltratadas por su pareja. 2011. [Consulta: 25 de agosto de 2018]. Disponible en: http://www.guiasalud.es/GPC/GPC 470 maltratadas_compl.pdf.

Sociedad EsPañola de GineCología y OBSTETRicica. Control Prenatal del embarazo normal Protocolo actualizado en Mayo de 2017. 2017. [Consulta: 1 de agosto de 2018]. Disponible en: https://bit.ly/33p19OL. 
STÖCKL, H. A move beyond screening is required to ensure adequate healthcare response for women who experience intimate partner violence. En: BMJ Evidence-Based Medicine. 2014, vol. 19, núm. 6, p. 240.

STÖCKL, H., et al. Acceptance of routine or case-based inquiry for intimate partner violence: a mixed method study. En: BMC Pregnancy and Childbirth. 2013, vol. 13, núm. 77, pp. 1-9.

TAYLOR, S.; BOGDAN, R. Introducción a los métodos cualitativos de investigación. La búsqueda de significados. Barcelona: Paidós, 1987.

TODAHL, J.; WAlters, E. Universal Screening for Intimate Partner Violence: a Systematic Rev. En: Journal of Marital and Family Therapy. 2011, vol. 37, núm. 3, pp. 355-369.

UNFPA - Fondo de Población de las Naciones Unidas; WAVE - NeTWORK AND European Info Centre against Violence. Strengthening Health System Responses to Gender- based Violence in Eastern Europe and Central Asia A Resource Package. 2014. [Consulta: 4 de agosto de 2019]. Disponible en: https://eeca.unfpa.org/sites/default/files/pub-pdf/WAVE-UNFPA-Report-EN.pdf.

UNIDAD DE IGUALDAD. UNIVERSIDAD DE MÁlAGA. Violencia de género en epoca de COVID-19. 2020. Disponible en: https://bit.ly/3I8qVmL.

VELASCO, C., et al. Intimate partner violence against Spanish pregnant women: Application of two screening instruments to assess prevalence and associated factors. En: Acta Obstetricia et Gynecologica Scandinavica. 2014, vol. 93, núm. 10, pp. 10501058.

WILliAMS, J. R., et al. An exploration of screening protocols for intimate partner violence in healthcare facilities: a qualitative study. En: Journal of Clinical Nursing. 2017, vol. 26, núms. 15-16, pp. 2192-2201. 